\title{
Hysteretic autoregressive time series models
}

\author{
By Guodong Li, Bo Guan, Wai Keung Li and Philip L.H. Yu \\ Department of Statistics and Actuarial Science, The University of Hong Kong, Pokfulam Road, \\ Hong Kong \\ gdli@hku.hk boyd.guan@gmail.com hrntlwk@hku.hk plhyu@hku.hk
}

\section{SUMMARY}

This paper extends the classical two-regime threshold autoregressive model by introducing hysteresis to its regime-switching structure, which leads to a new model: the hysteretic autoregressive model. The proposed model enjoys the piecewise linear structure of a threshold model but has a more flexible regime switching mechanism. A sufficient condition is given for geometric ergodicity. Conditional least squares estimation is discussed, and the asymptotic distributions of its estimators and information criteria for model selection are derived. Simulation results and an example support the model.

Some key words: Autoregression; Conditional least squares; Geometric ergodicity; Hysteresis; Threshold model.

\section{INTRODUCTION}

Threshold time series models have been very successful since their introduction by Tong (1978) and Tong \& Lim (1980); see also Chan (1993), Tsay (1998), Hansen (2000), and Ling \& Tong (2005). Due to its piecewise linear nature, the threshold model can mimic nonlinear features such as resonance, limit cycles and time-irreversibility, and it is also easy to fit numerically. Tong (1990) gives a comprehensive exposition of such models.

However, there is a sudden change in the probability structure when a threshold process switches regimes, which may not be the case in the real world. It has been observed that threshold models usually work well except around the boundaries between different regimes (Wu \& Chen, 2007). The smooth-transition threshold model (Chan \& Tong, 1986; van Dijk et al., 2002) can reduce this problem to some extent, but it may not function well in complicated cases, and it is not piecewise linear. Hamilton (1989) and McCulloch \& Tsay (1994) used the discrete-state Markov switching model to analyse financial and economic time series. Regime switching in that model is completely controlled by a latent random variable. The model enjoys some flexibility in the switching mechanism, but the fitted model may be difficult to interpret. Wu \& Chen (2007) considered a threshold variable-driven switching model in which the switching mechanism is jointly controlled by a latent variable and some observable variables, but this lacks a physical interpretation.

Hysteresis has been widely observed in economics, engineering, mechanics, material science, etc. Consider the simple microeconomic example in Gocke (2002). A previously inactive firm may have to bear a market entry cost to produce a certain product, i.e., production will become active only when the price of the product, $p_{t}$, is high enough to cover both the entry cost, $e_{t}$, and the cost of ingredients, $c_{t}$. However, once production has started, the firm will become inactive if and only if $p_{t}$ becomes too low to cover the cost $c_{t}$. As a result, when $c_{t}<p_{t}<c_{t}+e_{t}$, the active or inactive status remains unchanged. The original magnetic hysteresis of a single iron-crystal at 
40 the micro level exhibits exactly this pattern (Kneller, 1962), but hysteresis at the macro level has different and complicated patterns. All extant discussions of hysteresis in economics focus on the macro level, and there is no specific hysteretic model in econometrics and statistics. Camarero et al. (2006) and Proietti (2006) used non-stationarity to interpret hysteresis in macroeconomic time series, whilst Kannebley (2008) and Perez-Alonso \& Sanzo (2011) considered threshold 45 models.

From the viewpoint of threshold models, the hysteretic pattern at the micro level defines a regime switching mechanism, in which the active and inactive status correspond to two different regimes. This motivates us to propose a somewhat different type of piecewise linear model, which we call the hysteretic time series model. Consider a simple hysteretic process with hysteresis variable $z_{t}$ and hysteresis zone $\left(r_{L}, r_{U}\right]$. The time series is at the lower regime when $z_{t} \leq r_{L}$, and at the upper regime when $z_{t}>r_{U}$, and the regime remains unchanged as long as $z_{t}$ falls within the hysteresis zone. The Supplementary Material gives more details on the regime switching mechanisms of this model. This paper concentrates on the self-exciting hysteretic autoregressive model.

\section{HYSTERETIC AUTOREGRESSIVE PROCESSES}

Consider a self-exciting hysteretic autoregressive model with regime indicator $R_{t}$ :

$$
y_{t}=\left\{\begin{array}{l}
x_{t}^{\mathrm{T}} \phi+\sigma_{1} \varepsilon_{t}, R_{t}=1, \\
x_{t}^{\mathrm{T}} \psi+\sigma_{2} \varepsilon_{t}, R_{t}=0,
\end{array} \quad R_{t}= \begin{cases}1, & y_{t-d} \leq r_{L}, \\
0, & y_{t-d}>r_{U}, \\
R_{t-1}, & \text { otherwise },\end{cases}\right.
$$

where $x_{t}=\left(1, y_{t-1}, \ldots, y_{t-p}\right)^{\mathrm{T}}, \phi=\left(\gamma_{1}, \phi_{1}, \ldots, \phi_{p}\right)^{\mathrm{T}}, \psi=\left(\gamma_{2}, \psi_{1}, \ldots, \psi_{p}\right)^{\mathrm{T}}$, the $\varepsilon_{t}$ s are independent and identically distributed random variables with mean zero and variance one, $\sigma_{1}>0$ and $\sigma_{2}>0$ are scalars, integer $d>0$ is the delay parameter, and $r_{L} \leq r_{U}$ are the boundary parameters of the hysteresis zone. Model (1) includes the traditional two-regime threshold autoregressive model as a special case when $r_{L}=r_{U}$. The hysteretic model is also referred to as the buffered threshold model in Zhu et al. (2014).

From (1), the regime indicator takes the form

$$
\begin{aligned}
R_{t} & =I\left(y_{t-d} \leq r_{L}\right)+I\left(r_{L}<y_{t-d} \leq r_{U}\right) R_{t-1} \\
& =I\left(y_{t-d} \leq r_{L}\right)+\sum_{j=0}^{\infty} \prod_{i=0}^{j} I\left(r_{L}<y_{t-d-i} \leq r_{U}\right) I\left(y_{t-d-j-1} \leq r_{L}\right)
\end{aligned}
$$

almost surely. When $r_{L}<r_{U}$, the regime indicator $R_{t}$ depends on past observations that are infinitely far away, which renders the hysteretic model different from traditional threshold models (Tong, 1990; Hansen, 2000).

Suppose that $\sigma_{1}=\sigma_{2}=\sigma$. Denote $Y_{t}=\left(y_{t}, \ldots, y_{t-p+1}, R_{t}\right)^{\mathrm{T}}, e_{t}=\left(\sigma \varepsilon_{t}, 0, \ldots, 0\right)^{\mathrm{T}}, M_{0 t}=$ $\left(m_{0 t}, 0, \ldots, 0, I\left(y_{t-d} \leq r_{L}\right)\right)^{\mathrm{T}}$,

$$
M_{1 t}=\left(\begin{array}{ccccc}
m_{1 t} & m_{2 t} & \cdots & m_{p t} & 0 \\
1 & 0 & \cdots & 0 & 0 \\
\vdots & \vdots & \ddots & \vdots & \vdots \\
0 & 0 & \cdots & 0 & I\left(r_{L}<y_{t-d} \leq r_{U}\right)
\end{array}\right)
$$

$m_{0 t}=\gamma_{1} I\left(A_{t}\right)+\gamma_{2} I\left(A_{t}^{c}\right)$, and $m_{i t}=\phi_{i} I\left(A_{t}\right)+\psi_{i} I\left(A_{t}^{c}\right)$ with $i=1, \ldots, p$, where $A_{t}$ is the event $\left\{y_{t-d} \leq r_{L}\right\} \cup\left\{r_{L}<y_{t-d} \leq r_{U}, R_{t-1}=1\right\}$ and $A_{t}^{c}$ is its complement. It can be verified 
that $Y_{t}=g\left(Y_{t-1}\right)+e_{t}$ with $g\left(Y_{t-1}\right)=M_{0 t}+M_{1 t} Y_{t-1}$. As a result, $\left\{Y_{t}\right\}$ is a Markov chain, and, by a method similar to that in Chan \& Tong (1985), we can obtain its geometric ergodicity.

THEOREM 1. Suppose that $\varepsilon_{t}$ has a density function that is positive everywhere on $\mathbb{R}$ and $E\left(\left|\varepsilon_{t}\right|\right)<\infty$. If $\sum_{i=1}^{p}\left|\phi_{i}\right|<1$ and $\sum_{j=1}^{p}\left|\psi_{j}\right|<1$, then the multivariate process $\left\{Y_{t}\right\}$ is geometrically ergodic, and hence we can obtain the geometric ergodicity of the hysteretic process $\left\{y_{t}\right\}$.

The case with $\sigma_{1} \neq \sigma_{2}$ can be proved similarly. Chan \& Tong (1985) derived the geometric ergodicity of two-regime threshold autoregressive models under the same conditions as in Theorem 1.

\section{CONDITIONAL LEAST SQUARES ESTIMATION}

\subsection{Estimating procedure}

Let $\lambda=\left(\theta^{\mathrm{T}}, r_{L}, r_{U}, d\right)^{\mathrm{T}}$ denote the parameter vector of model $(1)$, where $\theta=\left(\phi^{\mathrm{T}}, \psi^{\mathrm{T}}\right)^{\mathrm{T}}$. Let $\Theta$ be a compact subset of $\mathbb{R}^{2 p+2},[a, b]$ be a predetermined interval and $d_{\max }$ be a predetermined positive integer. For the true values of parameter vector $\lambda$, we assume that $\theta_{0}=\left(\phi_{0}^{\mathrm{T}}, \psi_{0}^{\mathrm{T}}\right)^{\mathrm{T}}$ is an interior point of $\Theta, a<r_{0 L}<r_{0 U}<b$ and $d_{0} \in D=\left\{1, \ldots, d_{\max }\right\}$.

Denote the function for the sum of squared errors by $L_{n}(\lambda)=\sum_{t=1}^{n}\left\{\varepsilon_{t}(\lambda)\right\}^{2}$, where

$$
\varepsilon_{t}(\lambda)=\left(y_{t}-x_{t}^{\mathrm{T}} \phi\right) R_{t}\left(r_{L}, r_{U}, d\right)+\left(y_{t}-x_{t}^{\mathrm{T}} \psi\right)\left\{1-R_{t}\left(r_{L}, r_{U}, d\right)\right\} .
$$

Let $n_{0}=\max \left(p, d_{\max }\right)$. For time series $\left\{y_{t},-n_{0}+1 \leq t \leq n\right\}$ generated by model (1), the regime indicator function $R_{t}\left(r_{L}, r_{U}, d\right)$ in $\varepsilon_{t}(\lambda)$ depends on past observations that are infinitely far away, and hence initial values are needed for fitting.

For fixed $r_{L}, r_{U}$ and $d$, the first few observations of the hysteresis variable, say $y_{1-d}, \ldots, y_{t_{0}-d}$, may fall into the hysteresis zone $\left(r_{L}, r_{U}\right]$, such that we fail to identify the regimes of $y_{1}, \ldots, y_{t_{0}}$. We can simply assign them to the lower regime, and then denote the resulting regime indicator function by $\widetilde{R}_{t}\left(r_{L}, r_{U}, d\right)$. The exact value of $R_{t_{0}+1}\left(r_{L}, r_{U}, d\right)$ is known because $y_{t_{0}+1}$ lies outside the hysteresis zone, and it can be verified that $\widetilde{R}_{t}\left(r_{L}, r_{U}, d\right)=$ $R_{t}\left(r_{L}, r_{U}, d\right)$ as $t_{0}<t \leq n$.

By replacing $R_{t}\left(r_{L}, r_{U}, d\right)$ in the definition of $\varepsilon_{t}(\lambda)$ with $\widetilde{R}_{t}\left(r_{L}, r_{U}, d\right)$, we can denote the function $\widetilde{\varepsilon}_{t}(\lambda)$, and hence $\widetilde{L}_{n}(\lambda)$. Then the conditional least squares estimator can be defined as

$$
\widehat{\lambda}_{n}=\left(\widehat{\theta}_{n}^{\mathrm{T}}, \widehat{r}_{L}, \widehat{r}_{U}, \widehat{d}^{\mathrm{T}}=\underset{\lambda}{\operatorname{argmin}} \widetilde{L}_{n}(\lambda) .\right.
$$

Write $\widetilde{R}_{t}=\widetilde{R}_{t}\left(\widehat{r}_{L}, \widehat{r}_{U}, \widehat{d}\right)$ for simplicity. We further estimate $\sigma_{1}^{2}$ and $\sigma_{2}^{2}$ by $\widehat{\sigma}_{1 n}^{2}=$ $n_{1}^{-1} \sum_{t=1}^{n}\left(y_{t}-x_{t}^{\mathrm{T}} \widehat{\phi}_{n}\right)^{2} \widetilde{R}_{t}$ and $\widehat{\sigma}_{2 n}^{2}=n_{2}^{-1} \sum_{t=1}^{n}\left(y_{t}-x_{t}^{\mathrm{T}} \widehat{\psi}_{n}\right)^{2}\left(1-\widetilde{R}_{t}\right)$, respectively, where $\widehat{\theta}_{n}=\left(\widehat{\phi}_{n}^{\mathrm{T}}, \widehat{\psi}_{n}^{\mathrm{T}}\right)^{\mathrm{T}}, n_{1}=\sum_{t=1}^{n} \widetilde{R}_{t}$ and $n_{2}=n-n_{1}$.

Let $X_{t}=\left[x_{t}^{\mathrm{T}} \widetilde{R}_{t}\left(r_{L}, r_{U}, d\right), x_{t}^{\mathrm{T}}\left\{1-\widetilde{R}_{t}\left(r_{L}, r_{U}, d\right)\right\}\right]^{\mathrm{T}}$. For each fixed $\left(r_{L}, r_{U}, d\right)$, the minimiser of $\widetilde{L}_{n}(\lambda)$ has a closed form:

$$
\tilde{\theta}_{n}\left(r_{L}, r_{U}, d\right)=\underset{\theta}{\operatorname{argmin}} \widetilde{L}_{n}(\lambda)=\left(\sum_{t=1}^{n} X_{t} X_{t}^{\mathrm{T}}\right)^{-1} \sum_{t=1}^{n} X_{t} y_{t} .
$$

The step function $\widetilde{L}_{n}\left\{\widetilde{\theta}_{n}\left(r_{L}, r_{U}, d\right), r_{L}, r_{U}, d\right\}$ can be minimised by searching amongst all possible jumps, i.e. $d \in D$ and $\left(r_{L}, r_{U}\right) \in\left\{\left(y_{t-d}, y_{s-d}\right): 1 \leq t, s \leq n, a \leq y_{t-d} \leq y_{s-d} \leq b\right\}$; see $\operatorname{Li} \& \operatorname{Li}(2008,2011)$. 
For the initial values of the regime indicator function, we can instead assign these $t_{0}$ observations to the upper regime, and then denote the resulting function by $\widetilde{R}_{t}^{*}\left(r_{L}, r_{U}, d\right)$. Let $\widetilde{L}_{n}^{*}(\lambda)$ be the corresponding sum of squared errors, and $\widehat{\lambda}_{n}^{*}=\operatorname{argmin}_{\lambda} \widetilde{L}_{n}^{*}(\lambda)$. To improve accuracy, we can choose $\widehat{\lambda}_{n}^{*}$ as the conditional least squares estimator when $\widetilde{L}_{n}^{*}\left(\widehat{\lambda}_{n}^{*}\right)<\widetilde{L}_{n}\left(\widehat{\lambda}_{n}\right)$.

\subsection{Asymptotic results}

Assumption 1. It holds that $\phi_{0} \neq \psi_{0}, \operatorname{pr}\left(y_{t} \in[a, b]\right)<1$ and that $\varepsilon_{t}$ has a bounded, continuous and positive density on $\mathbb{R}$.

THEOREM 2. If the time series $\left\{y_{t}\right\}$ is strictly stationary and ergodic with $E\left(\left|y_{t}\right|^{2+\delta}\right)<\infty$ for a small $\delta>0$, and Assumption 1 holds, then $\widehat{\lambda}_{n} \rightarrow \lambda_{0}, \widehat{\sigma}_{1 n}^{2} \rightarrow \sigma_{01}^{2}$ and $\widehat{\sigma}_{2 n}^{2} \rightarrow \sigma_{02}^{2}$ almost surely, where $\lambda_{0}=\left(\theta_{0}^{\mathrm{T}}, r_{0 L}, r_{0 U}, d_{0}\right)^{\mathrm{T}}, \sigma_{01}^{2}$ and $\sigma_{02}^{2}$ are the corresponding true parameters.

We can prove Theorem 2 by standard arguments for strong consistency. The delay parameter $d$ takes only integer values, so $\widehat{d}$ will equal $d_{0}$ when sample size $n$ is sufficiently large. Without loss of generality, we assume that the true delay parameter, $d_{0}$, is known for the reminder of this subsection, and it is then deleted from parameter vector $\lambda$ and corresponding functions.

Assumption 2. The time series $\left\{y_{t}\right\}$ is strictly stationary with $E\left(y_{t}^{4+\delta}\right)<\infty$ for a small $\delta>0$, and $E\left(\varepsilon_{t}^{4}\right)<\infty$.

Assumption 3. The autoregressive function is discontinuous on the hysteresis zone $\left[r_{0 L}, r_{0 U}\right]$; i.e., there exist $p-1$ constants $z_{p-1}, \ldots, z_{p-d+1}, z_{p-d-1}, \ldots, z_{0}$, such that $z^{\mathrm{T}}\left(\psi_{0}-\phi_{0}\right) \neq 0$ for all $z_{p-d} \in\left[r_{0 L}, r_{0 U}\right]$, where $z=\left(1, z_{p-1}, \ldots, z_{0}\right)^{\mathrm{T}}$, and it is assumed that $d \leq p$ without loss of generality.

Let $Y_{t}=\left(y_{t}, \ldots, y_{t-p+1}, R_{t}\right)^{\mathrm{T}}$; then, from $\S 2,\left\{Y_{t}\right\}$ is a Markov chain. Denote its $m$-step transition probability function by $P^{m}(x, A)$, where $x \in \mathbb{R}^{p} \times\{0,1\}, A \in \mathcal{B}_{p} \times \mathcal{U}, \mathcal{B}_{p}$ is the class of Borel sets of $\mathbb{R}^{p}$, and $\mathcal{U}=\{\varnothing,\{0\},\{1\},\{0,1\}\}$.

Assumption 4. The time series $\left\{Y_{t}\right\}$ admits a unique invariant measure $\pi(\cdot)$, such that there exist $K>0$ and $0 \leq \rho<1$, for any $x \in \mathbb{R}^{p} \times\{0,1\}$ and any $m,\left\|P^{m}(x, \cdot)-\pi(\cdot)\right\|_{v} \leq K(1+$ $\|x\|) \rho^{m}$, where $\|\cdot\|_{v}$ and $\|\cdot\|$ are, respectively, the total variation norm and Euclidean norm.

Under Assumption 4, $\left\{Y_{t}\right\}$ is said to be $V$-uniformly ergodic with $V(x)=K(1+\|x\|)$, a condition stronger than geometric ergodicity; see Chapter 16 in Meyn \& Tweedie (1993). Following Chan \& Tong (1985) and Chan (1989), Assumption 4 is also implied by the conditions in Theorem 1.

THEOREM 3. If Assumptions 1-4 hold, then

(i) $n\left(\widehat{r}_{L}-r_{0 L}\right)=O_{p}(1), n\left(\widehat{r}_{U}-r_{0 U}\right)=O_{p}(1)$ and

(ii) $n^{1 / 2} \sup _{n\left(\left|r_{L}-r_{0 L}\right|+\left|r_{L}-r_{0 L}\right|\right) \leq B}\left\|\widetilde{\theta}_{n}\left(r_{L}, r_{U}\right)-\widetilde{\theta}_{n}\left(r_{0 L}, r_{0 U}\right)\right\|=o_{p}(1)$ for any fixed $0<B<$ $\infty$, where $\widetilde{\theta}_{n}\left(r_{L}, r_{U}\right)$ is defined as in (2).

140 Furthermore,

$$
n^{1 / 2}\left(\widehat{\theta}_{n}-\theta_{0}\right) \rightarrow N\left\{0, \operatorname{diag}\left(\sigma_{01}^{2} \Sigma_{1}^{-1}, \sigma_{02}^{2} \Sigma_{2}^{-1}\right)\right\}
$$

in distribution as $n \rightarrow \infty$, where $\Sigma_{1}=E\left(x_{t} x_{t}^{\mathrm{T}} R_{t}\right)$ and $\Sigma_{2}=E\left\{x_{t} x_{t}^{\mathrm{T}}\left(1-R_{t}\right)\right\}$.

Denote $\quad \xi_{1 t}=\sum_{j=0}^{\infty}\left[\left\{x_{t+j}^{\mathrm{T}}\left(\psi_{0}-\phi_{0}\right)\right\}^{2}+2 \sigma_{2} x_{t+j}^{\mathrm{T}}\left(\psi_{0}-\phi_{0}\right) \varepsilon_{t+j}\right] H_{t+j, j} \quad$ and $\quad \xi_{2 t}=$ $\sum_{j=0}^{\infty}\left[\left\{x_{t+j}^{\mathrm{T}}\left(\psi_{0}-\phi_{0}\right)\right\}^{2}-2 \sigma_{1} x_{t+j}^{\mathrm{T}}\left(\psi_{0}-\phi_{0}\right) \varepsilon_{t+j}\right] H_{t+j, j}, \quad$ where $\quad H_{t, j}=\prod_{l=1}^{j} I\left(r_{0 L}<\right.$ 
$\left.y_{t-d+1-l} \leq r_{0 U}\right)$ with the convention $\prod_{l=1}^{0}=1$. For $i=1$ and 2 , let $F_{i, L}(\cdot \mid r)$ and $F_{i, U}(\cdot \mid r)$ be the conditional distribution functions of $\xi_{i t}\left\{1-R_{t-1}\left(r_{0}\right)\right\}$ and $\xi_{i t} R_{t-1}\left(r_{0}\right)$ given $y_{t-d}=r$, respectively. Denote by $\pi(\cdot)$ the density function of $y_{t}$. We then define two independent one-dimensional two-sided compound Poisson processes:

$$
\wp_{L}(s)=I(s<0) \sum_{k=1}^{N_{1}^{(L)}(-s)} \zeta_{k}^{(1, L)}+I(s \geq 0) \sum_{k=1}^{N_{2}^{(L)}(-s)} \zeta_{k}^{(2, L)},
$$

and

$$
\wp_{U}(s)=I(s<0) \sum_{k=1}^{N_{1}^{(U)}(-s)} \zeta_{k}^{(1, U)}+I(s \geq 0) \sum_{k=1}^{N_{2}^{(U)}(-s)} \zeta_{k}^{(2, U)},
$$

where $\left\{N_{1}^{(L)}(s), s \geq 0\right\}$ and $\left\{N_{2}^{(L)}(s), s \geq 0\right\}$ are two Poisson processes with $N_{1}^{(L)}(0)=$ $N_{2}^{(L)}(0)=0$ and the same jump rate $\pi\left(r_{0 L}\right),\left\{N_{1}^{(U)}(s), s \geq 0\right\}$ and $\left\{N_{2}^{(U)}(s), s \geq 0\right\}$ are another two Poisson processes with $N_{1}^{(U)}(0)=N_{2}^{(U)}(0)=0$ and the same jump rate $\pi\left(r_{0 U}\right)$, $N_{1}^{(L)}(\cdot)$ and $N_{1}^{(U)}(\cdot)$ are left-continuous, $N_{2}^{(L)}(\cdot)$ and $N_{2}^{(U)}(\cdot)$ are right-continuous, and these four Poisson processes are independent. Each of the four sequences, $\left\{\zeta_{k}^{(i, j)}, k \geq 1\right\}$ with $i=1$ or 2 and $j=L$ or $U$, consists of independent and identically distributed random variables, where $\zeta_{k}^{(i, L)}$ and $\zeta_{k}^{(i, U)}$ have distribution functions $F_{i, L}\left(\cdot \mid r_{0 L}\right)$ and $F_{i, U}\left(\cdot \mid r_{0 U}\right)$, respectively.

For $j=L$ and $U$, Assumption 3 implies that $E\left(\zeta_{k}^{(1, j)}\right)=E\left(\zeta_{k}^{(2, j)}\right)>0$, and then $\wp_{j}(s)$ tends to $+\infty$ as $|s| \rightarrow \infty$. As in Li \& Ling (2012), there exists a unique random square $\left[M_{-}^{(L)}, M_{+}^{(L)}\right) \times$ $\left[M_{-}^{(U)}, M_{+}^{(U)}\right)$ on which the process $\wp(z)=\wp_{L}\left(z_{L}\right)+\wp_{U}\left(z_{U}\right)$ attains its global minimum, where $z=\left(z_{L}, z_{U}\right)^{\mathrm{T}} \in \mathbb{R}^{2}$ and $\left[M_{-}^{(j)}, M_{+}^{(j)}\right)=\operatorname{argmin}_{s \in \mathbb{R}} \wp_{j}(s)$ with $j=L$ or $U$.

THEOREM 4. If Assumptions 1-4 hold, then $n\left(\widehat{r}_{L}-r_{0 L}\right) \rightarrow M_{-}^{(L)}$ and $n\left(\widehat{r}_{U}-r_{0 U}\right) \rightarrow$ $M_{-}^{(U)}$ in distribution as $n \rightarrow \infty$. Moreover, $n\left(\widehat{r}_{L}-r_{0 L}\right), n\left(\widehat{r}_{U}-r_{0 U}\right)$ and $n^{1 / 2}\left(\widehat{\theta}_{n}-\theta_{0}\right)$ are asymptotically independent.

Theorem 4 can be obtained by combining the methods of proof for Theorem 3.3 in Li \& Ling (2012) and Theorem 2.3 in Li et al. (2013).

\subsection{Model selection}

To select the order $p$ of model (1), we consider the Bayesian information criterion,

$$
\operatorname{BIC}(p)=n_{1} \log \widehat{\sigma}_{1 n}^{2}+(p+1) \log n_{1}+n_{2} \log \widehat{\sigma}_{2 n}^{2}+(p+1) \log n_{2} .
$$

Similarly, we can define the Akaike information criterion, AIC, and the corrected AIC. Let $\widehat{p}_{n}=$ $\operatorname{argmin}_{0 \leq p \leq p_{\max }} \operatorname{BIC}(p)$, where $p_{\max }$ is a predetermined large order.

THEOREM 5. If $p_{\max } \geq p_{0}$ and Assumptions 1-4 hold, then $\operatorname{pr}\left(\widehat{p}_{n}=p_{0}\right) \rightarrow 1$ as $n \rightarrow \infty$, where $p_{0}$ is the true order, i.e. $\left|\phi_{0 p_{0}}\right|+\left|\psi_{0 p_{0}}\right|>0$.

We can show that minimisation of the AIC tends to select an order that is greater than or equal to $p_{0}$. Wong \& Li (1998) suggested information criteria for selecting the order of threshold models, and they are similar in form to (3). Moreover, we can consider different orders, say $p_{1}$ and $p_{2}$, for the two regimes of model (1) in the information criteria proposed above. 
Table 1. Percentages of correctly selected, over-fitted, under-fitted and wrongly selected models by the $\mathrm{AIC}$ and $\mathrm{BIC}$, respectively

\begin{tabular}{|c|c|c|c|c|c|c|c|c|}
\hline \multirow[b]{2}{*}{$n$} & \multicolumn{4}{|c|}{ AIC } & \multicolumn{4}{|c|}{ BIC } \\
\hline & Correct & Over & Under & Wrong & Correct & Over & Under & Wrong \\
\hline & \multicolumn{8}{|c|}{ Hysteretic autoregressive processes } \\
\hline 200 & $43 \cdot 0$ & $9 \cdot 2$ & $0 \cdot 3$ & $47 \cdot 5$ & $69 \cdot 2$ & $1 \cdot 3$ & $0 \cdot 7$ & $28 \cdot 8$ \\
\hline 400 & $70 \cdot 0$ & $12 \cdot 7$ & $0 \cdot 0$ & $17 \cdot 3$ & $95 \cdot 0$ & $0 \cdot 0$ & 1.0 & $4 \cdot 0$ \\
\hline \multirow[t]{2}{*}{800} & $87 \cdot 0$ & $12 \cdot 3$ & $0 \cdot 0$ & 0.7 & $100 \cdot 0$ & $0 \cdot 0$ & $0 \cdot 0$ & $0 \cdot 0$ \\
\hline & \multicolumn{8}{|c|}{ Threshold autoregressive processes } \\
\hline 200 & $83 \cdot 3$ & $15 \cdot 2$ & $1 \cdot 3$ & $0 \cdot 2$ & $86 \cdot 2$ & $12 \cdot 7$ & $0 \cdot 5$ & $0 \cdot 6$ \\
\hline 400 & $87 \cdot 5$ & $12 \cdot 5$ & $0 \cdot 0$ & $0 \cdot 0$ & $99 \cdot 0$ & $0 \cdot 3$ & $0 \cdot 7$ & $0 \cdot 0$ \\
\hline 800 & $91 \cdot 2$ & $8 \cdot 8$ & $0 \cdot 0$ & $0 \cdot 0$ & $100 \cdot 0$ & $0 \cdot 0$ & $0 \cdot 0$ & $0 \cdot 0$ \\
\hline
\end{tabular}

\subsection{Simulation experiment}

In an experiment to evaluate the information criteria in $\S 3.3$, two data-generating processes are employed. The first is a three-regime threshold autoregressive model,

$$
y_{t}=\left\{\begin{aligned}
-0.02 y_{t-1}+0 \cdot 76 y_{t-2}+\varepsilon_{t}, & y_{t-2} \leq-0 \cdot 9, \\
-0.70 y_{t-1}+0 \cdot 20 y_{t-2}+\varepsilon_{t}, & -0.9<y_{t-2} \leq 0 \cdot 5, \\
0.65 y_{t-1}+0.32 y_{t-2}+\varepsilon_{t}, & 0.5<y_{t-2},
\end{aligned}\right.
$$

and the second is a hysteretic autoregressive model with the same structure in the lower and upper regimes and the same delay and boundary parameters, where $\left\{\varepsilon_{t}\right\}$ are independent random variables with the standard normal distribution. These models both have two boundary or threshold parameters.

We consider sample sizes, $n=200,400$ and 800, with 1000 replications for each sample size and data-generating process. The hysteretic model and three-regime threshold model are both fitted to each generated series with $d_{\max }=4$, and the values of $a$ and $b$ are taken to be the 10th and 90th percentiles of each sample, respectively. The AIC and BIC are used in turn to select the model and order $p$ with $p_{\max }=4$. For the information criteria for the threshold model, see Wong \& Li (1998). Table 1 lists the percentages of correctly selected, over-fitted, under-fitted and wrongly selected models. Wrong selection refers to the case of a threshold model being selected for a hysteretic process or a hysteretic model being chosen for a threshold process. The percentages of correct selection increase for both the AIC and BIC as sample size $n$ increases, whilst the AIC has a slight tendency to select a bigger model even when $n=800$. A larger proportion of hysteretic processes is wrongly selected as threshold processes, particully when the sample size is as small as $n=200$. This may be because the hysteretic model includes the two-regime threshold model as a special case.

\subsection{Annual sunspot numbers}

The sequence of Wolf sunspot numbers is very popular in time series analysis, and various linear and nonlinear models have been applied to explore it. See, for example, the two-regime threshold models in Tong (1990) and the three-regime threshold models in Tsay (1989). Hysteresis has been observed in some solar activity cycles (Dmitriev et al., 2002; Suyal et al., 2012), and the sunspot number is a measure of solar activity. As a result, the hysteretic model may be more suitable for sunspot numbers than the threshold model.

We consider the annual mean Wolf sunspot numbers from 1700 to 2013, with 314 observations in total. The hysteretic autoregressive model is first applied to this sequence with $d_{\max }=6$, 
and the values of $a$ and $b$ are the 10th and 90th percentiles of the data, respectively. We allow the orders in both regimes to differ for the sake of parsimony. The AIC and BIC are employed to perform model selection with $p_{\max }=13$, and they choose the same model. Moreover, as a comparison, we consider two other classes of competing models, namely, autoregressive models and three-regime threshold autoregressive models. Both information criteria select the same autoregressive model but different threshold autoregressive models. As a result, a total of four models is selected.

The fitted delay parameter is $\widehat{d}=2$ for both the hysteretic and threshold models, which is consistent with the results in Tsay (1989). The Supplementary Material gives the sample autocorrelation functions of the residuals from these four fitted models, and we can conclude that they are all adequate. Their information criteria values, including those of the AIC, BIC and corrected AIC, are listed in Table 2. It can be seen that the hysteretic model outperforms all three other models, especially the autoregressive model.

Table 2. Information criteria of six fitted models

\begin{tabular}{ccccccc}
\hline & HAR & AR & TAR3-A & TAR3-B & TAR2-A & TAR2-B \\
\hline BIC & $1,568 \cdot 06$ & $1,692 \cdot 45$ & $1,610 \cdot 86$ & $1,588 \cdot 96$ & $1,577 \cdot 90$ & $1,575 \cdot 48$ \\
AIC & $1,512 \cdot 80$ & $1,651 \cdot 67$ & $1,522 \cdot 54$ & $1,529 \cdot 62$ & $1,522 \cdot 83$ & $1,526 \cdot 01$ \\
AICc & $1,820 \cdot 95$ & $1,955 \cdot 76$ & $1,842 \cdot 17$ & $1,842 \cdot 99$ & $1,831 \cdot 02$ & $1,833 \cdot 74$ \\
\hline
\end{tabular}

AICc, corrected AIC; AR, autoregressive model; HAR, hysteretic autoregressive model; TAR3-A and TAR3-B, three-regime threshold autoregressive model selected by the AIC and BIC, respectively; TAR2-A and TAR2-B, two-regime threshold autoregressive model selected by the AIC and BIC, respectively.

We also consider the class of two-regime threshold autoregressive models. Two models with different autoregressive orders are selected by the AIC and BIC. Their information criteria values are also given in Table 2. As expected, they are larger than the corresponding values of the fitted hysteretic model as the two-regime threshold model is a special case of the hysteretic model.

\section{ACKNOWLEDGEMENT}

We thank the editor, associate editor and two anonymous referees for comments that led to the substantial improvement of this paper, and acknowledge the Hong Kong Research Grants Council for partial support.

\section{SUPPLEMENTARY MATERIAL}

Supplementary material available at Biometrika online includes proofs of Theorems $1-5$, additional illustrations of the hysteretic models, more simulation results, more results on annual sunspot numbers and another example.

\section{REFERENCES}

Camarero, M., Carrion-I-Silvestre, J. L. \& TAMARit, C. (2006). Testing for hysteresis in unemployment in OECD countries: new evidence using stationarity panel tests with breaks. Oxford Bulletin of Economics and Statistics 68, 167-182.

CHAN, K. S. (1989). A note on the geometric ergodicity of a Markov chain. Advances in Applied Probability 21, $702-704$.

CHAN, K. S. (1993). Consistency and limiting distribution of the least squares estimator of a threshold autoregressive model. The Annals of Statistics 21, 520-533. 
CHAn, K. S. \& Tong, H. (1985). On the use of the deterministic Lyapunov function for the ergodicity of stochastic difference equations. Advances in Applied Probability 17, 666-678.

ChAn, K. S. \& Tong, H. (1986). On estimating thresholds in autoregressive models. Journal of Time Series Analysis 7, 178-190.

Dmitriev, A. V., Suvorova, A. V. \& Veselovsky, I. S. (2002). Expected hysteresis of the 23rd solar cycle in the heliosphere. Advances in Space Research 29, 475-479.

GocKE, M. (2002). Various concepts of hysteresis applied in economics. Journal of Economic Surveys 16, 167-188.

HAMILTON, J. (1989). A new approach to the economics analysis of nonstationary time series and the business cycle. Econometrica 57, 357-384.

HANSEN, B. E. (2000). Sample splitting and threshold estimation. Econometrica 68, 575-603.

KANNEBLEY, S. J. (2008). Tests for the hysteresis hypothesis in Brazilian industrialized exports: a threshold cointegration analysis. Economic Modelling 25, 171-190.

250 KNELLER, E. (1962). Ferromagnetismus. Berlin: Springer.

LI, D. \& LING, S. (2012). On the least squares estimation of multiple-regime threshold autoregressive models. Journal of Econometrics 167, 240-253.

LI, D., LING, S. \& LI, W. K. (2013). Asymptotic theory on the least squares estimation of threshold moving-average models. Econometric Theory 29, 482-516.

LI, G. \& LI, W. K. (2008). Testing for threshold moving average with conditional heteroscedasticity. Statistica Sinica 18, 647-665.

LI, G. \& LI, W. K. (2011). Testing a linear time series model against its threshold extension. Biometrika 98, 243-250.

Ling, S. \& Tong, H. (2005). Testing for a linear MA model against threshold MA models. The Annals of Statistics 33, 2529-2552.

MCCUlloch, R. \& TSAY, R. S. (1994). Statistical analysis of economic time series via Markov switching models. Journal of Time Series Analysis 15, 235-250.

Meyn, S. P. \& Tweedie, R. L. (1993). Markov Chains and Stochastic Stability. New York: Springer.

Perez-Alonso, A. \& SANZO, S. D. (2011). Unemployment and hysteresis: a nonlinear unobserved components approach. Studies in Nonlinear Dynamics and Econometrics 15, 1-27.

Proietti, T. (2006). Trend-cycle decompositions with correlated components. Econometric Reviews 25, 61-84.

SuYAl, V., Prasad, A. \& Singh, H. P. (2012). Hysteresis in a solar activity cycle. Solar Physics 276, 407-414.

Tong, H. (1978). On a threshold model. In Pattern Recognition and Signal Processing, C. H. Chen, ed. Amsterdam: Sijthoff and Noordhoff, pp. 575-586.

Tong, H. (1990). Nonlinear Time Series: A Dynamical System Approach. Oxford: Oxford University Press.

Tong, H. \& LIM, K. S. (1980). Threshold autoregression, limit cycles and cyclical data (with discussion). Journal of the Royal Statistical Society, Series B 42, 245-292.

TSAY, R. S. (1989). Testing and modeling threshold autoregressive processes. Journal of the American Statistical Association 84, 231-240.

275 TSAY, R. S. (1998). Testing and modeling multivariate threshold models. Journal of the American Statistical Association 93, 1188-1202.

VAn DiJK, D., TerasvirTa, T. \& Franses, P. H. (2002). Smooth transition autoregressive models - a survey of recent developments. Econometric Reviews 21, 1-47.

WonG, C. S. \& LI, W. K. (1998). A note on the corrected Akaike information criterion for threshold autoregressive models. Journal of Time Series Analysis 19, 113-124.

WU, S. \& CHEN, R. (2007). Threshold variable determination and threshold variable driven switching autoregressive models. Statistica Sinica 17, 241-264.

ZHU, K., YU, P. L. H. \& LI, W. K. (2014). Testing for the buffered autoregressive processes. Statistica Sinica 24, 971-984. 